\title{
"Zhejiang Experience": Problems and Countermeasures in the Construction of Internet Courts*
}

\author{
Hanying Zhu \\ China Jiliang University \\ Hangzhou, China
}

\begin{abstract}
The Internet Court of Hangzhou has been in existence for more than one year. In the process of construction and development, the results of the practice have been very fruitful, also has problems exposed. As the first Internet court in the country, Hangzhou Internet Court has a unique position and superiority. It analyzes the problems and countermeasures generated during the construction process, and then summarizes the construction experience, which helps to build the "Zhejiang sample" of the Internet court, make recommendations for the realization of the goal of "smart court" in Zhejiang Province, and promotes the "Internet and Judicial" system in China.
\end{abstract}

Keywords-internet court; online litigation; artificial intelligence

\section{INTRODUCTION}

The trial mode of the "Internet case for Internet trials" by the Hangzhou Internet Court has yielded remarkable results and greatly promoted the pace of the construction of the "Smart Court" in Zhejiang Province. The quality of trials has improved significantly and has been widely recognized by all sectors of society. They use artificial intelligence technology to develop intelligent trial systems, realize the intelligence from the filing to the referee, and promote institutional innovation, forming a "six platforms, three models and one system" Internet court construction template. It provides experience for the promotion of Internet courts nationwide and exploring new rules for litigation and refereeing in Internet cases.

\section{II. "SiX PlatForms, ThreE MODELS AND ONE} SYSTEM": A SUCCESSFUL MODEL OF ZHEJIANG EXPERIENCE

\section{A. Six Platforms: Integrated Construction of Online Litigation Service Platform}

Through the establishment of six integrated online litigation service platforms, Hangzhou Internet Court has improved online litigation services and achieved the reform

*Project: Zhejiang Experience, Problems and Countermeasures in the Construction of Internet Courts

Item Number: 2019221103560Bi00001 goal of "no need to run once".

The online litigation platform is the basis and starting point for the litigants to file online litigation; the online mediation platform provides online mediation services for the litigants. The electronic evidence platform standardizes the format, access, transmission and access of electronic evidence, and solidifies it while generating electronic data to ensure the credibility and authenticity of electronic evidence. The electronic delivery platform greatly improves the efficiency of delivery, litigants can receive legal documents in a timely manner; effectively solve the problem of delivery. The online execution platform provides technical support for online execution, and relies on Internet technology to greatly improve execution efficiency and ease the difficulty of implementation. The trial "big data" platform provides data references for judges and litigants. These six platforms are effectively connecting each link of the network trial. They run through the whole process of the network-related case trials, and become the basis for the development and the improvement of the online litigation mode of the Internet court.

\section{B. Innovation: Artificial Intelligence Responds to the Needs of the Judicial Era}

The Hangzhou Internet Court embodies the in-depth application of artificial intelligence technology in the judicial field and is the epitome of the construction of the "smart court" in Zhejiang Province. Hangzhou Internet Court is very innovative and forward-looking to integrate artificial intelligence technology with judicial litigation system. It not only provides technical support for realizing the network operation mode of Internet court litigation, but also greatly improves the case handling efficiency. It facilitates the litigants, opening up a new model of judicial reform, and showing a new form of justice and convenience.

By using the artificial intelligence case handling system, the court can preliminarily harmonize the standards and rules for evidence identification. It can access electronic evidence online to ensure the authenticity and reliability of electronic evidence, and also realize the automatic operation of the trial system and synchronize the intelligent generation of electronic files, thus further improving the intelligence level in the trial process of the Internet court. 


\section{Deepening: Improving Internet Litigation Mode of the Joint Market Depository Platform}

Internet courts should cooperate with social organizations such as Internet companies, electronic depository institutions, and universities in a proactive manner. They can coconstruct the most efficient and reliable online trial mode, improve the effectiveness of trials, and optimize the Internet judicial litigation system.

The Hangzhou Internet Court signed cooperation agreements with some of the People's Mediation Committee of Internet companies such as Tencent, JD.COM and Sogou to jointly explore a diversified dispute resolution mechanism for Internet cases.

They also cooperate with third-party depository platforms such as Ancun, CFCA, and E-sign ${ }^{1}$, promote the construction of electronic evidence platforms, and establish rules for the identification of electronic evidence. At the same time, they cooperated with Ant Financial, officially launched the judicial block chain, and explored the best mode of judicial block chain application. They also signed a strategic cooperation agreement with Zhejiang Sci-Tech University to realize resource sharing, jointly cultivate talents, promote the construction and development of Internet courts, and improve the online litigation mechanism.

\section{Think tank: Establishing Internet Law Research Institute and Building an Intellectual Support Platform}

The Internet Law Institute is a high-end think tank that studies leading issues in the field of Internet law and provides decision-making advice and solutions for the formulation of relevant laws and policies and the proper resolution of emerging Internet cases in judicial practice. It has absorbed a group of outstanding university research scholars and experts in the Internet practice community to conduct comprehensive research and applied research on the Internet justice field and promote the process of Internet rule of law construction. The Internet Law Institute has an important position as a "think tank" in the process of building Internet courts. It will not only help solve Internet governance problems, but also provide ideas for exploring new modes of Internet justice, and contribute enough wisdom to Internet governance.

\section{E. Results: Significant Improvement in Trial Quality}

By the end of December 2018, the Hangzhou Internet Court had accepted 15,456 cases and concluded 13,604 cases. The average court time for online related cases was 28 minutes, and the average court time and trial period were $66.8 \%$ and $25 \%$ lower than the traditional model. The online trial and online referee rate is $60 \%$, the service rate is up to $97.8 \%$, and the automatic fulfillment rate of the litigants is

E-signing company is the originator of China's third-party electronic signature platform. It is the first company to enter the electronic evidence platform of Hangzhou Internet Court to realize direct communication of electronic data. At the same time, it is connected to the notary office, the judicial appraisal center and other relevant judicial institutions, using blockchain technology to achieve data synchronization and notarization and storage.
97\%. Internet court judges closed 905 cases per capita, ranking first in the province, and the quality of trials was significantly improved. ${ }^{2}$

TABLE I. COMPARISON OF THE EVALUATION OF THE QUALITY OF TRIALS BETWEEN THE COURTS IN HANGZHOU AND THE INTERNET COURTS

\begin{tabular}{|c|c|c|}
\hline \multirow[b]{2}{*}{$\begin{array}{c}\text { Trial quality } \\
\text { evaluation index } \\
\text { (as of the end of 2018) }\end{array}$} & \multicolumn{2}{|c|}{ Reasonable interval } \\
\hline & $\begin{array}{l}\text { The average } \\
\text { value of the } \\
\text { citywide court in } \\
\text { Hangzhou }\end{array}$ & $\begin{array}{c}\text { Hangzhou } \\
\text { Internet Court }\end{array}$ \\
\hline Average court time & 54 minutes & 18 minutes \\
\hline $\begin{array}{c}\text { Number of cases closed } \\
\text { by per capita judges }\end{array}$ & 427.7 (pieces) & 905 (pieces) \\
\hline $\begin{array}{c}\text { Total number of cases } \\
\text { accepted }\end{array}$ & 331439 (pieces) & 15456(pieces) \\
\hline $\begin{array}{c}\text { Total number of cases } \\
\text { concluded }\end{array}$ & 347023(pieces) & 13604(pieces) \\
\hline Average trial days (days) & 49.75 days & 37.31 days \\
\hline $\begin{array}{l}\text { Civil mediation and } \\
\text { withdrawal rate (\%) }\end{array}$ & $21.9 \%$ & $96.9 \%$ \\
\hline
\end{tabular}

\section{RISKS AND DIFFICULTIES: PROBLEMS FACED BY THE INTERNET COURT}

\section{A. The Innovation of the Litigation System Under the Online Trial Mode Is Not Compatible with the Rules of the Litigation Law}

The online trial mode of the Internet court makes the whole process of the case process networked. Therefore, procedures, such as accepting cases, determining the qualifications of litigants, and obtaining and using evidence are different from the traditional offline mode. The rules for the regulation of the process and specific links of the traditional offline mode cannot be fully applied to the online trial mode of the Internet court, and conflicts will arise in the specific application process. The application of the online trial mode under these conflicts has raised the issue of the rationality of electronic delivery, the application of electronic evidence, the rationality of the proceedings in the second instance, and the negative impact on the litigants' right to appeal. Although the Hangzhou Internet Court has tentatively proposed a solution, the basic and principled standards involved in some issues still need to be improved from the system design and legislative level, and provide direction for the further development of the Internet judicial system.

\section{B. The Exploration of Cross-regional Jurisdiction Is Not Thorough Enough}

The terms of the agreement not only provide a legal basis for the Internet court to accept more cross-regional and

\footnotetext{
2 The data comes from the "Work Report of Hangzhou Intermediate People's Court (Abstract)", "Hangzhou Daily", the fourth edition of January 29, 2019.

Referring to the evaluation data of Hangzhou Intermediate People's Court from 2017 to 2018, the project team members set a corresponding interval. From http://hangzhou.zjcourt.cn/col/col1218409/index.html, last visit time: April
} $1,2019$. 
foreign-related cases, but also create conditions for the Hangzhou Internet Court to accumulate foreign-related trial experience and actively explore new modes and new experiences of Internet justice. However, most of the cases handled by the Hangzhou Internet Court in practice are cases involving the geographical location of Hangzhou, and the number of cases across regions and foreign countries is small. The cross-regional trials of the Internet Court in Hangzhou are not thorough enough. They have not fully utilized the characteristics of "Internet + Judicature" to break away from space constraints and explore the experience of cross-border transnational case trials. Due to the special "pilot" nature of the Hangzhou Internet Court and the uniqueness of the online trial model, the types of cases under its jurisdiction should be more cumbersome, and the scope of the case should be more extensive, let it draw more trial and practice experience, and be able to better play the sample role of the first Internet court in the country.

\section{The Development of Internet Technology Brings Risks and Challenges to Online Trials}

The Internet courts have a high degree of reliance on network information technology. From the case to the conclusion, all the links are networked. The online trial mode can only be realized by relying on Internet technology. However, the high risk, unpredictability and easy change of Internet technology may cause security risks on the platform. For example, Internet technology can achieve falsification or even destruction of electronic evidence, intrusion into the internal data information system of the court, and the illegal purpose of interfering with trials. Moreover, in the process of transmission of relevant trial information, business secrets, personal privacy and state secrets may be involved. In the network trial, the protection of the privacy of the litigants, the security of the case information, the fairness and authority of the judicial trial are threatened. How to ensure information security? This is an important challenge for Internet courts. In addition, Internet technology updates are faster, and Internet courts need a certain amount of time to adapt to the new network environment. The establishment of relevant legal rules is not a one-step process. Therefore, the adaptation of the Internet court online trial mode faces technological changes and challenges.

\section{A Relatively Short Shortage of Specialized Talents}

The shortage of talent teams in the Hangzhou Internet Court is mainly reflected in the fact that the number of judges is small and the reserve talents are insufficient. The large number of cases accepted by the Hangzhou Internet Court is the main reason for the shortage of specialized talents. According to the data of 2018, there are 905 cases in which the Internet Court judges in Hangzhou have ended the trial, that is, each judges have to complete 2.5 cases on average every day. Moreover, the Hangzhou Internet Court and the original Hangzhou Railway Transport Court used the same group of people. In the case of a surge in Internetrelated cases, the pressure on the judges was inevitably aggravated. Due to the special nature of Internet courts in the type of case jurisdiction and litigation process, traditional legal talents have insufficient understanding of this new trial mode, and it is difficult for them to adapt to this model in the short term. In practice, they must be trained to be competent in the courts. Therefore, it is necessary to cultivate professional Internet legal talents and expand the number of judges.

\section{MECHANISM: COUNTERMEASURES FOR THE CONSTRUCTION OF INTERNET COURTS}

\section{A. Improving Laws and Regulations to Improve the Litigation Rules of Network-related Cases, and Promoting the Formulation of Electronic Litigation Law}

Legislation should be used to determine the principles and standards of online trials, and to improve the litigation rules for network-related cases. Optimize rules that conflict with current procedural law provisions. On the basis of following the rules of the procedural law, it's necessary to respect the uniqueness of the trial mode and comprehensively consider establishing legal and reasonable litigation rules. It is necessary to respect the subjective will of the litigants, establish a unified, complete and systematic electronic delivery specification, and clarify the electronic delivery workflow and specifications to ensure the rationality of electronic delivery. Establish rules and standards for the application of electronic evidence ensure the authenticity and integrity of electronic evidence, and ensure fairness and justice to solve problems arising from the application of electronic evidence. On the basis of complying with the provisions of the procedural law, supplementary provisions should be made for the litigation process of the Internetrelated appeal cases. Furthermore, under the current environment driven by electronic communication and network interaction, the electronic litigation law is urgently needed to improve and ensure the orderly and fair resolution of disputes involving networks. A new set of special legislation in the field of electronic litigation should be established to systematically regulate online trial mode and network environment.

\section{B. Promoting Cross-regional Agreement Jurisdiction}

The innovation of the jurisdictional system and the expansion of jurisdiction are the core features of the Internet court. However, in the current practice, the Hangzhou Internet Court did not fully utilize this advantage to explore trial experience. To this end, the Hangzhou Internet Court should clearly define its own position, actively carry out foreign exchanges and cooperation, and increase the international recognition and popularity of Internet courts; this will attract more litigants involved in foreign-related cases to agree to the Hangzhou Internet Court as a court of jurisdiction and to accumulate experience in cross-regional network-related cases. At the same time, the Hangzhou Internet Court should also maximize its own advantages. Under the premise of not violating the provisions of the procedural law on jurisdiction, it should actively explore the trial experience of cross-regional and cross-border networkrelated cases, and provide opinions for the trial of such cases to fully realize the innovation of jurisdiction. 


\section{Using Technology and Big Data Platforms to Prevent Data Security Risks}

In order to ensure the security of online trials and to circumvent possible risks, Internet courts must improve their technical level and comprehensive strength. First, it is necessary to strengthen the sense of safety responsibility of judges and staff to ensure that relevant confidential information is not leaked, and the legitimate rights and interests of the litigants are not damaged. Second, it is to cooperate with domestic and foreign first-class technology companies to improve litigation platforms and the technical system of online trials. The security, stability, and reliability of all aspects of the network litigation process should be guaranteed. Further improve the digital encryption technology to ensure the authenticity and integrity of electronic documents and files. The third is to keep up with the direction of big data development and ensure the stability and convenience of storage data information interaction. With the help of big data platform, the exchange and sharing of data information will be strengthened to ensure the authenticity of data sources. Through the above points, it's necessary to improve the construction of technology platforms and avoid the risks brought by the application of Internet technologies.

\section{Strengthening the Construction of the Talent Team in the Internet Court and Improving the Training Mechanism for Internet Court Judges}

First, it is necessary to appropriately expand the ranks of judges and attract professional and outstanding talents. On this basis, a group of judges with high professional theoretical literacy and high-level practical trial ability will be trained. Actively invite experts and scholars in the field of Internet and law to conduct regular professional training for judges and conduct learning forums to enable judges to keep pace with the times and improve their overall quality.

The second is to cooperate with major university law schools to cultivate counterpart talents, and the two sides jointly establish a talent training base. Guide students to understand the Internet Court by setting up relevant internship positions within the court, organizing students to visit the courts, and organizing relevant training courses. Expand the talent team of the Internet court and improve the trial quality and trial efficiency of the Internet court.

\section{CONCLUSION}

The Zhejiang judicial system carried forward the "red boat spirit" proposed by President Xi Jinping, integrated the latest technology to establish the Hangzhou Internet Court. Through these actions, we can sum up a series of Zhejiang experience. The main problems and challenges faced by the Internet court, such as the incompatibility between the litigation rules and the current litigation system, the insufficient exploration of the case jurisdiction system, the large data security risks in the litigation platform, and the shortage of talents, can be solved by the following methods: promoting the development of electronic litigation law, cross-regional agreement jurisdiction, multi-dimensional construction of data safety net, strengthening the construction of talent team. Looking forward to the future development of the Internet court, we must build a "Zhejiang sample" of Internet court construction, further expand the types of cases involving Internet cases, promote AI trials, and establish unmanned courts. This is an important way to obtain an international voice in the Internet referee. In terms of international cyberspace governance, providing Chinese programs and Chinese wisdom has become a more profound value of this project.

\section{REFERENCES}

[1] Chen Guangzhong. Evidence Law [M]. Beijing: Law Press, 2015.

[2] Gao Fuping. Research on China's e-commerce legislation [M]. Beijing: Law Press, 2015

[3] (US) Marcela et al. Network forensics: from data to electronic evidence [M]. Beijing: Chinese People's Public Security University Press, 2015.

[4] Mi Zhenrong. Essentials of Internet Disputes [M]. Beijing: People's Court Press, 2018.

[5] Hou Meng. The Impact of Internet Technology on Judicature - A Case Study of Hangzhou Internet Court [J]. The law applies, 2018(1).

[6] Hiroyuki Masuyama.Continuous-time block-monotone Markov chains and their block-augmented truncations.Linear algebra and its applications, 13 November, 2015

[7] Tania Sourdin,Archie Zariski. What Is Responsive Judging Springer Singapore, 2018.

[8] Lin William Cong. Zhiguo He.Blockchain Disruption and Smart Contracts.Economics of Networks eJournal, 14 Jun, 2017. 